\title{
The positive side of the coin: Sars-Cov-2 pandemic has taught us how much Telemedicine is useful as standard of care procedure in real life
}

\author{
Khadija El Aoufy ${ }^{1}$ - Maria Ramona Melis ${ }^{1}$. Silvia Bellando Randone ${ }^{1}$. Jelena Blagojevic ${ }^{1}$. Francesca Bartoli ${ }^{1}$. \\ Ginevra Fiori ${ }^{1}$. Francesca Nacci ${ }^{1}$. Maria Letizia Conforti ${ }^{1} \cdot$ Laura Cometi $^{1}$ - Cosimo Bruni ${ }^{1}$. Martina Orlandi ${ }^{1}$. \\ Alberto Moggi-Pignone ${ }^{2} \cdot$ Laura Rasero $^{3} \cdot$ Serena Guiducci ${ }^{1} \cdot$ Marco Matucci-Cerinic $^{1,4}$
}

Received: 30 August 2021 / Revised: 7 October 2021 / Accepted: 23 October 2021 / Published online: 5 November 2021

(c) International League of Associations for Rheumatology (ILAR) 2021

\begin{abstract}
Patients and health workers were at high risk of infection during the Sars-Cov-2 pandemic lockdown. For this reason, other medical and clinical approaches such as Telemedicine were necessary. Despite Telemedicine was born before COVID-19, the pandemic was the opportunity to accelerate a process already underway for at least a decade and to blow all the barriers away. Our aim is to describe the experience of Telemedicine during and immediately after the first lockdown to assure the follow-up in a 'virtual' outpatient clinic dedicated to Rheumatic and Musculoskeletal Diseases (RMDs) and to give an overview of Telemedicine in the rheumatology field. We retrospectively evaluated the patient flow to our rheumatology division from March to September 2020 and, in accordance with local restrictions, three periods were selected. In the 1st period, 96.96\% of the outpatient clinic cases were shifted to Telemedicine; these decreased to $52.45 \%$ in the 2 nd period, while the 3rd period was characterized by the return of the patients at the clinic $(97.6 \%)$. Diagnostic procedures were postponed during the 1 st period, reduced drastically during the 2 nd and performed regularly during the third period. Intravenous infusions were maintained as much as possible during the three periods, to assure therapeutic continuity. Shifting stable patients to Telemedicine has the potential to allow continuity of care, while reducing the risk of contagion during a pandemic. In the next future, the integration of Telemedicine as standard of care for specific clinical applications might assure assistance for RMDs patients also in non-pandemic conditions.
\end{abstract}

Keywords COVID-19 $\cdot$ Rheumatic and Musculoskeletal Diseases $\cdot$ Sars-Cov-2 $\cdot$ Telemedicine

Khadija El Aoufy

khadija.ela92@gmail.com; khadija.elaoufy@unifi.it

1 Department of Experimental and Clinical Medicine, \& Department of Geriatric Medicine, Division of Rheumatology AOUC, University of Florence, Viale Largo BrambillaFirenze (FI), 3 - 50134 Florence, Italy

2 Department of Experimental and Clinical Medicine, \& Department of Emergency Medicine DEA, Division of Internal Medicine AOUC, University of Florence, Florence, Italy

3 Department of Public Health AOUC, University of Florence, Florence, Italy

4 Unit of Immunology, Rheumatology, Allergy and Rare Diseases (UnIRAR), IRCCS San Raffaele Hospital, Milan, Italy

\section{Introduction}

\section{The Sars-Cov-2 pandemic and Rheumatic and Musculoskeletal Diseases (RMDs)}

In the last 2 years, the COVID-19 has put at the stake the majority of healthcare systems. In particular, the lockdown has imposed worldwide unprecedented restrictions to the clinical standard care of RMDs patients. In Italy, most of the routine activities were cancelled during the streaming of the pandemic in March 2020, prompting a pragmatic reorganization of the traditional clinical activities and care model, thus providing a rapid and efficient response to patients' needs.

In the literature, the effects of the lockdown on RMDs patients are now documented [1-4]. In fact, the restrictions significantly affected the emotional well-being and favoured the disease relapse [5]. Both these two items have 
been approached with Telemedicine to screen severe symptoms in real time, despite the lockdown. In other healthcare settings, Telemedicine was implemented in response to the pandemic, providing a high patient and provider satisfaction [6]. In particular, Telemedicine represented a valid and useful addition to the follow-ups of rheumatic patients. This evidence suggests that Telemedicine may serve as a new approach for the follow-up of patients with RMDs [7-9]. Consequently, Telemedicine and Healthcare Technologies have clearly become a smart opportunity for the care and management of RMDs to achieve and maintain high standards of quality care during an emergency. All these experiences should lay the foundations for a procedural habit to integrate in everyday practice.

\section{Telemedicine and its use in clinical care: definition and purpose}

In 2010, WHO (World Health Organization) has defined Telemedicine as a big opportunity: 'the delivery of health care services, where distance is a critical factor, by all health care professionals using information and communication technologies for the exchange of valid information for diagnosis, treatment and prevention of disease and injuries, research and evaluation, and for the continuing education of health care providers, all in the interests of advancing the health of individuals and their communities' [9]. Indeed, Telemedicine was born before COVID-19, yet the pandemic accelerated a process already underway for at least a decade. In the first place, Telemedicine was employed to ensure access to healthcare in rural areas, to easily obtain health services remaining within their local community [10]. Then, Telemedicine has been widely employed in chronic diseases. In Italy, the COVID-19 crisis determined a rapid technological expansion not only for healthcare but also for students attending school lessons, and for the population to maintain relationships with relatives and friends [7].

During the COVID-19 outbreak, Telemedicine has provided a basic medical support to the community, including RMDs patients. Telemedicine also helped patients not to remain isolated and has protected patients and physicians from contact, therefore possibly reducing the chance of cross-infection in hospitals, eliminating social panic, enhancing the public's self-protection ability, correcting improper medical treatment behaviour and promoting epidemiological screening [7]. Despite its large use, some procedures still await standardization, while several issues such as patient privacy protection, medical insurance, expense reimbursement, data security and doctor's remuneration still need to be addressed $[11,12]$.

\section{Ethics in the act of telemedicine}

Ethical considerations are mandatory to deal with, when talking about telemedicine, to guarantee a safer use of the services. The ethical aspects of telemedicine are widely analysed in the literature, indeed, most authors have strongly stressed out the protection of patient information, confidentiality, informed consent, physician's malpractice and lack of specific regulations [13].

As reported by Langarizadeh et al., the ethical issues in telemedicine can be investigated from several aspects, including technology, doctor-patient relationship, data confidentiality and security, informed consent, patient's and family's satisfaction with telemedicine services [14].

Moreover, Nittari et al. stated that the use of telemedicine may potentially overcome any kind of organizational and practical deficit. Despite this, it would be a priority to preventively develop and ameliorate those actual shortcomings, in order to assure useful and vital activities for the patient [15].

In our context, regional laws were applied providing a web-based platform to perform the Telemedicine services, in which patients were identified through their Fiscal Code.

\section{Remuneration of the medical consultation}

The essential objective of e-health is to contribute to the reorganization of the healthcare system, rationalizing and optimizing the current models, while improving overall efficiency and reducing management costs. Currently, Telemedicine consultations are remunerated according to regional/ national regulations, which make them equal to traditional consultations in terms of costs.

In the meantime, a National Study Group on the Economic Evaluation of Telemedicine Services has been established by the Italian Institute of Public Health (ISS) to specifically define cost/effectiveness evaluation models for these services and identify new pricing systems. Moreover, Interim indications for telemedicine assistance services during the COVID-19 health emergency were activated. Numerous variables should be taken into consideration when dealing with the reimbursement of telematic services, such as the technological capacity, the public or private nature and the location of the structures in which these are provided, as well as maintenance costs, the possible reduction of waiting times and of working days loss [16].

\section{Strengths and weaknesses of Telemedicine}

We know that Telemedicine allows the continuity of care and follow-ups in time, thus supporting the patients at distance. 
This entails real benefits for the health system, in terms of reduction in waiting times and limiting the risk of infection (see Table 1). Moreover, patients were satisfied to maintain the contact with the physician and to reduce the travelling costs and the loss of working days as well [4].

During this period of crisis, patients' compliance to treatment was a critical issue and the risk of treatment interruption was perceived as a main problem. Thus, switching to a Telemedicine-based hybrid model was likely to maintain as much as possible drug compliance, with substantial savings on loss of pay and out-of-pocket expenditure [17].

Some weaknesses of Telemedicine are obvious, like the inability to perform a physical and joint examination and the limited access to the web, in particular for elderly patients. Some patients also showed a psychological and cultural reluctance for online consultations because they preferred the face-to-face visit. Likewise, Telemedicine is not appropriate for patients failing to respond to therapies (see Table 1) [17-19]. In fact, new signs/symptoms and disease flare should not be limited to the Telemedicine approach.

It is likely that the impetuous growth of telemedicine that has occurred in many countries will be confirmed and consolidated even when the pandemic is finally over. Despite this, the majority of healthcare systems neither provided the tools (PC, Phones, cameras, etc.), nor funds and standardized protocols or reimbursement policy to support Telemedicine [17-19]. These limitations might have impacted on the use of Telemedicine despite the pandemic advancement and should be overcome to carefully integrate Telemedicine as a standard of care procedure in the future.

\section{Telemedicine in rheumatology: telerheumatology}

The pandemic impacted remarkably on rheumatology practice, from the access to outpatient clinic, to the hospitalization, and even in prescribing anchor drugs. In fact, the restricted access to rheumatology care jeopardized the control of chronic RMDs and their long-term prognosis [19].

In Arab countries, COVID-19 pandemic determines on average $65 \%$ decrease in outpatient consultations,
$56 \%$ decrease in day hospital-infusion centres and a $69 \%$ decrease in regular hospitalization, both lowest in the Gulf and highest in North Africa [19]. The rheumatologists interviewed reported using Telemedicine in $70 \%$ of the cases, mostly based on traditional telephone contacts and e-mails, and to be reimbursed in $12 \%$ of the cases.

Before the pandemic, other studies supported the use of Telemedicine in rheumatology: a tele-monitoring service was implemented in 2012 to deliver healthcare to patients with stable RA and SpA in an outpatient clinic in Singapore [20]. The service was steered by two advanced practice nurses once a week, with up to 50 patients/per month.

During the pandemic, Telemedicine has increased from 10 to $90 \%$ of patient contacts in only 1 week. In Australia, a remarkable success was obtained with tele-rheumatology for up to $80 \%$ of outpatient appointments [21], while a cross-sectional study conducted in April 2020 in Puducherry (India) reported that $76.1 \%$ out of 373 RMDs patients in conditions of poverty considered tele-rheumatology better than in-person consultation. The teleconsultations were limited to telephone calls and photographs shared through WhatsApp [22].

Recently, a positive experience with telephone and video consultations in Italian SLE and psoriatic arthritis patients was reported [23]. From March 11, 2020, all patients affected by RMDs and treated with biological disease-modifying anti-rheumatic drugs afferent to a Rheumatology Unit (Catania) were contacted to evaluate the health status, laboratory test reports and the presence of any adverse events. A nurse administered the clinimetric questionnaires to evaluate the disease activity and the impact of RMDs on the health status.

According to a recent systematic review, Telemedicine was well-accepted by RMDs patients and rheumatologists, while remote consultations were unsuitable for new patients, the elderly, those with language barriers and for patients with only one annual visit [24].

In the Netherlands, a survey among rheumatologists indicated that the missed personal interaction was felt as an unmet need, although less travel time, ease of use of the

Table 1 Aspects of disease manageable and not manageable with telemedicine

\begin{tabular}{ll}
\hline Aspects of RMDs manageable with Telemedicine & Aspects of RMDs not fully manageable with Telemedicine \\
\hline - Continued patient care during pandemic events & • First consultation of complex cases \\
- Regular follow up for stable patients & - Physical/joint examination \\
- Patient education & - Thorough evaluation of disease flare \\
- Clinimetry/Telemetry & - Management of digital lesions \\
- Patient counseling & \\
- Detection of urgent problems & \\
- Drug Adherence and patients' compliance & \\
- Detection of drug-related adverse effects & \\
- Dealing with general practitioners & \\
- Renewal of the therapeutic prescriptions &
\end{tabular}


system and shorter waiting period were indicated as the 3 key elements for patients to favour telemedicine [18].

During the first period of the pandemic, $17 \%$ of 548 worldwide rheumatologists reported that at least a quarter of their patients might not have access to telehealth contacts because of connections issues, particularly those below the poverty line $[25,26]$.

Clearly, all these topics should be considered when approaching the use of Telemedicine. Guidelines and recommendations are in fact urgently needed in the field of rheumatology. Today, it is evident that Telemedicine should be managed not only by well-prepared clinicians and Healthcare Professionals (HPs), but also specific guidelines and national rules are urgently needed to guide the development of this emergent area.

\section{Evidence obtained by our experience}

In our centre, outpatient visits dropped by more than $60 \%$ during the first phase of the pandemic. This reality forcefully prompted the shift to Telemedicine to assure continuity of care with follow-ups at distance for RMDs patients. Data from our Rheumatology division show the pivotal contribution of Telemedicine during the lockdown which tightly restricted the access to the outpatient clinic. The report of our experience was evaluated by the local Ethical Committee (Comitato Etico Area Vasta Centro, Toscana, Italy) and approval was waived because no clinical or demographic data concerning the patients was analysed in the study.

At the beginning of the pandemic, a team of rheumatologists and nurses was set up to revise and study, on a weekly basis, each patients' charts to plan the
Telemedicine sessions. The physical or virtual presence of the patient in the clinic was decided case-by-case and adapted to the patient's needs and preferences, considering also the clinical picture previously discussed by the rheumatology team.

The patients' flow to the outpatient 'virtual' Telemedicine clinic dedicated to RMDs during and immediately after the lockdown was divided in 3 periods according to national indications. Indeed, an almost complete restriction of the access to the hospital was maintained from March 9th to May 18th (1st period) and in the following period up to June the 30th (when limitations of the access to health services were still maintained - 2nd period). During the 3 rd period, from June the 30th up to September the 30th, 2020, there were no specific restrictions on the access to healthcare facilities.

From March to September 2020, the number of scheduled infusions performed were 653/913 (71.5\%) in the first, $542 / 542(100 \%)$ in the second and $1.048 / 1.048(100 \%)$ in the third period.

In the outpatient clinic, the shift to Telemedicine was done in $96.96 \%$ of the cases during the 1 st period. This number decreased to $52.45 \%$ in the 2 nd period, while $97.6 \%$ of the consultations were carried out in the 3rd period. Diagnostic procedures, such as ultrasound, capillaroscopy, and joint injection were generally postponed during the 1 st period, reduced drastically during the 2 nd and performed regularly during the 3 rd period. Ulcer treatment and the Clinical Trial Unit never stopped their activity. The trend of visits and Telemedicine divided per month is shown in Fig. 1. We observe that Telemedicine increased most in the first period and gradually diminished in the second period, while in the third period, no Telemedicine was activated.

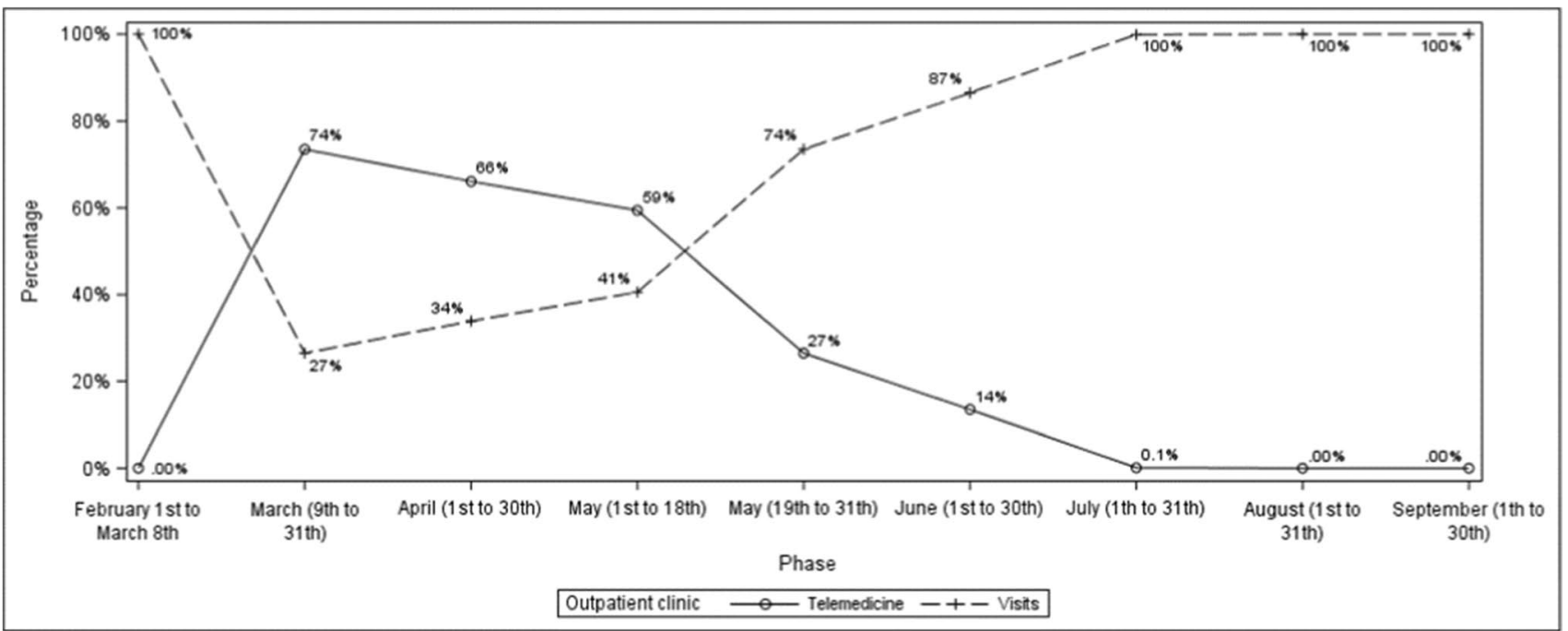

Fig. 1 The percentage of visits and Telemedicine during the SARS-CoV-2 pandemic from February 2020 to September 2020 
Indeed, the last period is characterized by medical examination and full access to the centre (Fig. 1).

The reorganization of patient care is the most challenging aspect of adaptation to the pandemic. This may vary depending on local policy, hospital/clinic organization, volume of clinical activity and available facilities/staff [27]. In addition, it is important that patients are adequately informed about COVID-19 signs and symptoms. Our strategy was to assure that patients were adequately informed about COVID-19 manifestations and hygienic rules of prevention, maintaining an optimal communication and cooperation between rheumatologists, nurses and general practitioners [28, 29]. In our cohort, we successfully performed remote laboratory monitoring, as well as switching from intravenous to subcutaneous drug administration and from nurse-led group teaching of subcutaneous drug administration to online teaching supported by written information and video demonstrations. This was possible despite the rapid increase of the pandemic and Telemedicine follow-up consultations for RMDs patients during the $1^{\text {st }}$ and $2^{\text {nd }}$ periods. The evaluation of clinical symptoms and blood tests, as well as drug prescription and psychological support, were the main activities. Conversely, in-person appointments were always guaranteed and prioritized to discuss difficult news related to disease severity, still providing, when possible, the option of telephone or video conference for virtual follow-up.

In this light, at least part of other routine follow-up visits may easily be adapted to Telemedicine, saving unnecessary travel and still assuring patient care [20]. For what concerns patients with ulcers and related complications, it is important to underline how their constant access to the centre despite the pandemic risks has taught us that this kind of clinical issue is difficult to treat at distance and almost always must be directly taken care of. Indeed, for ulcer management and patient education in an outpatient setting, the contribution of Telemedicine should be expanded but still limited despite the fact that it may grossly help through ulcer monitoring by photos or videos. For instance, the patient might need to consult the specialist nurse to provide high-quality care advices and guidance.

Our data show that Telemedicine can be helpful mainly in some areas of activity of a rheumatology division, like the outpatient clinic, where in the future it could be employed also in 'normal' conditions for a tight follow-up of stable patients. In our opinion, patients with RMDs are an optimal population target for Telemedicine, as they are usually younger people of working age, with a probably active lifestyle and children. On the other hand, also the elderly or limited-mobility population may benefit from remote visits, allowing greater freedom for the caregiver.

As the biological therapy has changed the progression of these diseases, favouring periods of remission, remote consultations through Telemedicine may lighten the burden of the disease with more frequent follow-ups. Obviously, the first rheumatology consultation for RMDs patients must still be face-to-face because Telemedicine cannot fully replace the standard clinical approach [30]. Consequently, virtual appointments are the key to reducing stable patients access to healthcare services and preventing the risk of infection, during a pandemic emergency, limiting the exposure bidirectionally both for patients and healthcare providers, still providing a fast track of access for unstable patients with complications and saving unnecessary travel [31].

\section{Conclusions}

The pandemic experience has taught us that we need not only to educate the physicians to optimize their Telemedicine approach but also to increase the number of specialized nurses in rheumatology. The healthcare systems should take valuable lessons from these unprecedented events, while rheumatologists and HPs should promptly shape together new strategies with the wise use of healthcare technologies. In the next months, up to the end of 2021, the experience previously accumulated [26, 30,32-35] will be relevant for the healthcare systems expecting an other potential wave. In fact, Telemedicine should be maintained and gradually included as a dichotomous standard of care tool either in pandemic and standard conditions. Stable or in-remission patients are preferred for a Telemedicine approach, while other cases should be considered for a face-to-face visit.

In conclusion, our experience has taught us that the continuity of patient care is mandatory in clinical practice, and its interruption is not affordable even in a pandemic situation. Also, Telemedicine should be further implemented in the divisions of rheumatology worldwide in the next future.

Acknowledgements The project (Telereuma) has been supported by an unrestricted grant of Biogen, BMS and Novartis.

\section{Declarations}

Disclosures None.

\section{References}

1. Cankurtaran D, Tezel N, Ercan B, Yildiz SY, Akyuz EU (2021) The effects of COVID-19 fear and anxiety on symptom severity, sleep quality, and mood in patients with fibromyalgia: a pilot study. Adv Rheumatol 61(1):41. https://doi.org/10.1186/ s42358-021-00200-9

2. Gupta L, Kharbanda R, Agarwal V, Misra DP, Agarwal V (2021) Patient perspectives on the effect of the SARS-CoV-2 pandemic on patients with systemic sclerosis: an international patient survey. J Clin Rheumatol 27(1):31-33. https://doi.org/10.1097/RHU. 0000000000001681 
3. Ammitzbøll C, Andersen JB, Vils SR, Mistegaard CE, Mikkelsen $S$ et al (2021) Isolation, behavioral changes and low seroprevalence of SARS-CoV-2 antibodies in patients with Systemic Lupus Erythematosus or Rheumatoid arthritis. Arthritis Care Res (Hoboken). https://doi.org/10.1002/acr.24716

4. Garrido-Cumbrera M, Marzo-Ortega H, Christen L, PlazueloRamos P, Webb D et al (2021) Assessment of impact of the COVID-19 pandemic from the perspective of patients with rheumatic and musculoskeletal diseases in Europe: results from the REUMAVID study (phase 1). RMD Open 7(1):e001546. https://doi.org/10.1136/rmdopen-2020-001546

5. Iannuccelli C, Lucchino B, Gioia C, Dolcini G, Favretti M, Franculli D, Di Franco M (2021) Mental health and well-being during the COVID-19 pandemic: stress vulnerability, resilience and mood disturbances in fibromyalgia and rheumatoid arthritis. Clin Exp Rheumatol 39 Suppl 130(3):153-160

6. Morales-Torres J, Aceves-Ávila FJ (2020) Rheumatologists in the COVID-19 era: will there be a new role for the rheumatologist in the care of rheumatic patients? Clin Rheumatol 39(11):3177-3183. https://doi.org/10.1007/ s10067-020-05380-1

7. Webster P (2020) Virtual health care in the era of COVID-19. Lancet 395(10231):1180-1181. https://doi.org/10.1016/S01406736(20)30818-7

8. Xie T, Wang D, Wang X, Yang Q, Sun H, Liu R, Li M (2021) Impact of COVID-19 pandemic on outpatient appointments of rheumatic patients in a non-outbreak area of China. Wien Klin Wochenschr 18:1-10. https://doi.org/10.1007/ s00508-021-01891-2

9. WHO Global Observatory for eHealth (2010) Telemedicine: opportunities and developments in Member States: report on the second global survey on eHealth. World Health Organization https://www.who.int/goe/publications/goe_telemedicine_ 2010.pdf

10. Akintayo RO, Akpabio AA, Kalla AA, Dey D, Migowa AN, Olaosebikan $\mathrm{H}$ et al (2021) The impact of COVID-19 on rheumatology practice across Africa. Rheumatology (Oxford) 60(1):392-398. https://doi.org/10.1093/rheumatology/keaa600

11. Matsumoto RA, Barton JL (2021) Telerheumatology: before, during, and after a global pandemic. Curr Opin Rheumatol 33(3):262-269. https://doi.org/10.1097/BOR.0000000000 000790

12. Bateman J, Cleaton N (2021) Managing patients using telerheumatology: lessons from a pandemic. Best Pract Res Clin Rheumatol 35(1):101662. https://doi.org/10.1016/j.berh.2021. 101662

13. Chaet D, Clearfield R, Sabin JE, Skimming K, Council on Ethical and Judicial Affairs American Medical Association (2017) Ethical practice in Telehealth and Telemedicine. J Gen Intern Med 32(10):1136-1140. https://doi.org/10.1007/ s11606-017-4082-2

14. Langarizadeh M, Moghbeli F, Aliabadi A (2017) Application of ethics for providing telemedicine services and information technology. Med Arch 71(5):351-355. https://doi.org/10.5455/ medarh.2017.71.351-355

15. Nittari G, Khuman R, Baldoni S, Pallotta G, Battineni $G$ et al (2020) Telemedicine practice: review of the current ethical and legal challenges. Telemed J E Health 26(12):1427-1437. https:// doi.org/10.1089/tmj.2019.0158

16. Gabbrielli et al. In ISS COVID-19 Report, no. 12/2020- https:// www.iss.it/documents/20126/0/Rapporto+ISS+COVID-19+n.+ 12_2020+telemedicina.pdf/387420ca-0b5d-ab65-b60d-9fa42 $6 \mathrm{~d} 2 \mathrm{~b} 2 \mathrm{c} 7 ? \mathrm{t}=1587114370414$

17. Bonfá E, Gossec L, Isenberg DA, Li Z, Raychaudhuri S (2021) How COVID-19 is changing rheumatology clinical practice.
Nat Rev Rheumatol 17(1):11-15. https://doi.org/10.1038/ s41584-020-00527-5

18. Bos HW, Van Tuberger A, Vonkeman AE et al (2020) Telemedicine for patients with rheumatic and musculoskeletal diseases during the COVID-19 pandemic; a positive experience in the Netherlands. Rheumatol Int. https://doi.org/10.1007/ s00296-020-04771-6

19. Ziade $\mathrm{N}$ et al (2020) The impact of COVID-19 pandemic on rheumatology practice: a cross-sectional multinational study. Clin Rheumatol. https://doi.org/10.1007/s10067-020-05428-2

20. Chew LC, Yeo SI, Thomboo J et al (2020) The impact of the offsite monitoring clinic (virtual monitoring clinic) on the practice of outpatient rheumatology in a tertiary centre during the COVID-19 pandemic. Ann Acad Med Singap 49:905-8. https:// doi.org/10.47102/annals-acadmedsg.2020222

21. Kulcsar Z, Albert D, Ercolano E, Mecchella JN (2016) Telerheumatology: a technology appropriate for virtually all. Semin Arthritis Rheum 46(3):380-385. https://doi.org/10.1016/j.semar thrit.2016.05.013

22. Kavadichanda C, Shah S, Daber A et al (2020) Tele-rheumatology for overcoming socioeconomic barriers to healthcare in resource constrained settings: lessons from COVID-19 pandemic. Rheumatology 00:1-11. https://doi.org/10.1093/rheum atology/keaa791

23. Foti R, Amato G, Visalli E et al (2020) Management of patients with inflammatory rheumatic diseases: telemedicine and rheumatologists challenged in the era of COVID-19. Front Public Health 8:558838. https://doi.org/10.3389/fpubh.2020.558838

24. Piga M, Cangemi I, Mathieu A, Cauli A (2017) Telemedicine for patients with rheumatic diseases: systematic review and proposal for research agenda. Semin Arthritis Rheum 47:121-128. https://doi.org/10.1016/j.semarthrit.2017.03.014

25. Metha B, Jannat Khaha D, Fontana MA et al (2020) Impact of COVID-19 on vulnerable patients with rheumatic disease: results of a worldwide survey. RMD Open 6:e001378. https:// doi.org/10.1136/rmdopen-2020-001378

26. Kernder A, Morf H, Klemm P, Vossen D, Haase I, Mucke J et al (2021) Digital rheumatology in the era of COVID-19: results of a national patient and physician survey. RMD Open 7(1):e001548. https://doi.org/10.1136/rmdopen-2020-001548

27 Rawaf S, Allen LN, Stigler FL, Kringos D, Quezada Yamamoto H, van Weel C (2020) Global forum on universal health coverage and primary health care. Lessons on the COVID-19 pandemic, for and by primary care professionals worldwide. Eur J Gen Pract. 26(1):129-133. https://doi.org/10.1080/13814788. 2020.1820479

28. WHO Coronavirus Disease (COVID-19) Dashboard [Internet]. [cited 3 December 2020]. https://covid19.who.int.

29. Romão VC, Cordeiro I, Macieira C, Oliveira-Ramos F, Romeu JC, Rosa CM, Saavedra MJ et al (2020) Rheumatology practice amidst the COVID-19 pandemic: a pragmatic view. RMD Open 6(2):e001314. https://doi.org/10.1136/rmdopen-2020-001314

30. Howren A, Aviña-Zubieta JA, Rebić N, Dau H, Gastonguay L, Shojania K et al (2020) Virtual rheumatology appointments during the COVID-19 pandemic: an international survey of perspectives of patients with rheumatic diseases. Clin Rheumatol 39(11):3191-3193. https://doi.org/10.1007/ s10067-020-05338-3

31. Moazzami B, Razavi-Khorasani N, Dooghaie Moghadam A, Farokhi E, Rezaei N (2020) COVID-19 and telemedicine: immediate action required for maintaining healthcare providers well-being. J Clin Virol 126:104345. https://doi.org/10.1016/j. jcv.2020.104345

32. Koonin LM, Hoots B, Tsang CA, Leroy Z, Farris K, Jolly T et al (2020) Trends in the use of telehealth during the emergence of the COVID-19 pandemic - United States. MMWR Morb Mortal 
Wkly Rep 30;69(43):1595-1599. https://doi.org/10.15585/ mmwr.mm6943a3

33. Hollander JE, Carr GC (2020) Virtually perfect? Telemedicine for Covid-19. N Engl J Med 382(18):1679-2168. https://doi. org/10.1056/NEJMp2003539

34. Cronin O, Horne A, Ralston SH (2020) Rheumatology in a time of Coronavirus: lessons from our early experiences. QJM 113(10):715-716. https://doi.org/10.1093/qjmed/hcaa163

35. McDougall JA, Ferucci ED, Glover J et al (2017) Telerheumatology: a systematic review. Arthritis Care Res (Hoboken) 69(10):1546-1557. https://doi.org/10.1002/acr.23153
Publisher's note Springer Nature remains neutral with regard to jurisdictional claims in published maps and institutional affiliations. 